\begin{tabular}{|c|c|c|}
\hline PORT SAID ENGINEERING RESEARCH JOURNAL & Faculty of Engineering - Port Said University \\
(Production Engineering \& Mechanical Design)
\end{tabular}

\title{
Influence of Adjusting Moment of Inertia on Behavior of Electromagnetic Energy Harvester
}

\author{
Shahenda M. Attya ${ }^{1}$, Ebtisam F. Abd-Gwad. ${ }^{2}$ \\ Received: 8 January 2020; Accepted: 26 April 2020
}

\begin{abstract}
Recently, with the urgent need for renewable and clean energy sources, the emphasis has been on extracting and generating energy from the surrounding environment. Harvesting mechanical kinetic energy is an attractive field for easy access to energy from multiple sources, and transforming it out of the mechanical domain to the electrical domain. Resonant systems introduced a magic solution in maximizing the output power when the ambient frequency is similar to the natural frequency of the mechanism. But the main drawback of these systems remains when moving away from the resonance zone even slightly, where that leads to a significant reduction in the output power. This paper discusses the design of an innovative autonomous mechanism for tuning the resonant frequency and thereby generate larger amounts of energy. Unlike previous researches which rely heavily on tuning the resonant frequency by changing the spring stiffness, the proposed concept is based on adjusting the resonant frequency by automatically modifying the mass moment of inertia. The proposed energy harvester used the electromagnetic technique to transform mechanical vibration into electric power. This design is analyzed using the methodology of a vibration continuous system. Different parameters of the tuning mechanism have been investigated to achieve the resonant. The tuned mechanism has been examined experimentally and then validated by comparing experimental results with the results obtained through the analytical model.
\end{abstract}

Keywords: Vibration energy harvester, Tuning, Resonant, Electromagnetic

\section{INTRODUCTION}

Latterly, a lot of researches concentrated on energy harvesting by tuning the resonant frequency. The tuning technique relies on modifying the system's natural frequency until it reaches the ambient frequency and as a result, the system remains in a resonant state. The most amount of energy can be harvested when the system reaches the resonance state. Resonant frequency tuning can be performed either manually or autonomously. Manual tuning requires an external operator whilst autonomous tuning is a tuning mechanism performed by the harvester itself without human intervention. In Autonomous tuning, closed-loop control is requisite for tuning the resonant frequency at all times[1]. Dibin Zhu [2] provided a review of the most important research on different tuning methodologies. Sutrisno W. and Wahied G. [3] also presented a useful review of the most common

\footnotetext{
${ }^{1}$ Assistant Lecturer, Department of Production Engineering and Mechanical Design, Faculty of Engineering, Port Said University, Egypt, email: eng_sh_2007@eng.psu.edu.eg

2 Professor, Department of Production Engineering and

Mechanical Design, Faculty of Engineering, Port Said University, Egypt, email: ebtisam1953@yahoo.com

DOI: $10.21608 /$ pserj.2020.22167.1028
}

frequency tuning methods used for piezoelectric energy harvesters.

Resonant frequency tuning can be classified according to its methodology into two types, the first one is electrical tuning whilst the other is mechanical tuning. Electrical methods usually depend on modifying the electrical loads of the harvester for tuning the resonance frequency. The mechanical tuning methods include adding a tip mass, modifying the dimensions of the structure, adjusting the center of gravity of proof mass, and changing the spring stiffness.

Whereas the mechanism mass is a fundamental parameter affecting the natural frequency, Cornwell et al. [4] tuned the resonant frequency by adding an auxiliary structure, and the prototype frequency was tuned from $40.7 \mathrm{HZ}$ to $38.2 \mathrm{HZ}$. This improved the output power from the system, which was harvested using a piezoelectric harvester. Miller et al.[5] improved this approach for (MEMS) piezoelectric energy harvester this harvester connected to an integrated thick film electrochemical micro capacitor. the pneumatic dispenser is utilized for depositing a tip mass and a capacitor to realize the resonant state. The study was able to adjust the resonant frequency from $102 \mathrm{HZ}$ to 82 HZ with tunability $21.74 \%$ and sensitivity $25 \mathrm{~Hz} / \mathrm{mm}$. Actually, when an energy harvester is manufactured, it is difficult to add or remove an effective mass to it, and 
therefore the resonant frequency of it can be tuned by repositioning its centroid. Wu et al. [6] have tuned the natural frequency of piezoelectric energy harvester by adding a movable mass (steel stud) which is manually moved to change the centroid mechanism position. The resonant frequency of the proposed mechanism was from 130 to $180 \mathrm{~Hz}$ with tunability $32.26 \%$. The drawback of this approach is that it ignored the influence of the shape and dimensions of the proof mass during analyzing the proposed model, and these parameters have been considered ineffective. Schaufuss et al. [7] developed this design by using a lever mechanism instead of the traditional mass and spring system. The position of the proof mass was modified manually by a screw. The mechanism natural frequency can be tuned in the range from 42 to $55 \mathrm{HZ}$ with the tunability of about $26.8 \%$ and sensitivity $0.26 \mathrm{HZ} / \mathrm{mm}$.

The concept of the proposed design depends on tuning the resonance frequency by adjusting the moment of inertia of the tuning mechanism and this by changing the position of the proof mass automatically and thus, the natural frequency of the tuning mechanism matches with the ambient frequency whatever the change in frequency.

The most common model that represents many of the vibratory energy harvesters is the second-order springmass system with a single degree of freedom. Williams and Yates [8] illustrate the basic components of this system which include a frame that transmits the vibration to an inertial mass via a spring and a damper for achieving a relative displacement or strain. This basic model is only suitable for linear harvesters, where mechanical damping and stiffness are proportional to speed and displacement respectively.

Therefore, this study focused on analyzing the proposed prototype using the methodology of a vibration continuous system. The theoretical model of the cantilever beam energy harvester is used to anticipate its natural frequency value while changing the tuning mass position and its mass moment of inertia as a result. The dynamic response of the ambient harmonic vibration and the power harvested can be also simulated theoretically. The proposed design is investigated experimentally to validate the results of the analytical model.

\section{DYNAMIC MODEL}

\subsection{Free Vibration Model}

The proposed energy harvester in Figure (2) is analyzed using the methodology of a vibration continuous system, and it is solved with the MATLAB program. The administering equation of the transverse motion can be given by applying the Euler-Bernoulli beam theory and Newton's low in the transverse direction $[9,10]$.

$\rho A \frac{\partial^{2} w(x, t)}{\partial t^{2}}+E I \frac{\partial^{4} w(x, t)}{\partial x^{4}}=F(x, t)$
Where $w(x, t)$ represents the cantilever transverse deflection at position $(x)$ and time $(t),(\mathrm{E})$ is the cantilever modulus of elasticity, ( $\rho$ ) is the density, (A) is the cantilever cross-section area, (I) is the cantilever second moment of area, and $F(x, t)$ is the externally applied force whereas $F(x, t)=0$ concerning free vibration.
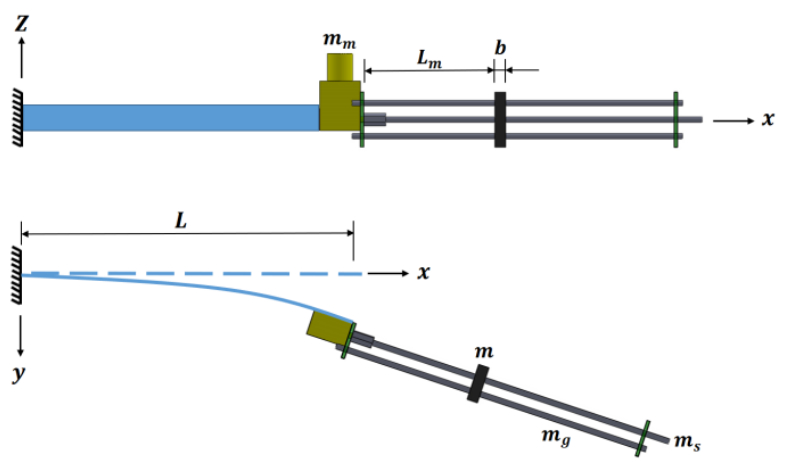

Figure 1 Schematic diagram of the proposed design parameters

The general solution of the previous equation can be found using the separation of a variable method as:

$T(t)=A \sin \omega_{n} t+B \cos \omega_{n} t$

$W(x)=c_{1} \sin \beta_{n} x+c_{2} \cos \beta_{n} x+c_{3} \sinh \beta_{n} x+$

$$
c_{4} \cosh \beta_{n} x
$$

Where $\beta_{n}^{4}=\frac{\rho A \omega_{n}^{2}}{E I}$

$\mathrm{W}(\mathrm{x})$ is known as the cantilever beam transverse deflection at position $(\mathrm{x}),\left(\omega_{n}\right)$ is the natural frequency of the proposed mechanism. Since each continuous system contains an infinite number of natural frequencies and normal modes, this study was conducted by focusing on the first natural frequency. The constants (A) and (B) can be determined from the initial conditions and constants $\left(c_{1}, c_{2}, c_{3}\right.$, and $\left.c_{4}\right)$ can be obtained from the following boundary conditions.

a) At the fixed end of the cantilever beam at $(x=0)$ the transverse deflection and its slope are zero.

$W(0)=C_{2}+C_{4}=0$

$\frac{d W(0)}{d x}=C_{1}+C_{3}=0$

b) At the other free end of the cantilever beam at ( $x=$ $L)$

- A shear force is equal to the inertia force due to the total mass $\left(M_{t}\right)$ attached to the tip of the cantilever beam and the damping force.

$$
\begin{aligned}
& I E \frac{\partial^{3} w(L, t)}{\partial x^{3}}=M_{t} \frac{\partial^{2} w(L, t)}{\partial t^{2}}+C_{t} \frac{\partial w(L, t)}{\partial t} \\
& M_{t}=m+2 m_{g}+m_{s}+m_{m}+m_{a}
\end{aligned}
$$


Where $(m)$ is the movable tuning mass, $\left(m_{g}\right)$ is the mass of the guide shafts, $\left(m_{s}\right)$ is the screw mass, $\left(m_{m}\right)$ is DC motor mass, and $\left(m_{a}\right)$ is a mass of the acrylic frame.

When studying the effect of damping on the analyzed system, three types of damping have appeared:

- Electromagnetic damping $\left(C_{m}\right)$ caused by the electromagnetic force.

- Structural damping $\left(C_{s}\right)$ caused by the internal friction of the cantilever.

- air-viscous damping $\left(C_{a}\right)$ due to air particles' resistance to vibratory cantilever movement.

Thus total damping coefficient $\left(C_{t}\right)$ is the sum of these three damping coefficients.

And by substituting equation (3) into equation (6), the equation becomes:

$C_{1}\left[-I E \beta_{1}^{3} \cos \beta_{1} L+\left(M_{t} \omega_{1}^{2}-C_{t} \omega_{1}\right) \sin \beta_{1} L\right]+$ $C_{2}\left[I E \beta_{1}^{3} \sin \beta_{1} L+\left(M_{t} \omega_{1}^{2}-C_{t} \omega_{1}\right) \cos \beta_{1} L\right]+$ $C_{3}\left[I E \beta_{1}^{3} \cosh \beta_{1} L+\left(M_{t} \omega_{1}^{2}-C_{t} \omega_{1}\right) \sinh \beta_{1} L\right]+$ $C_{4}\left[I E \beta_{1}^{3} \sinh \beta_{1} L+\left(M_{t} \omega_{1}^{2}-C_{t} \omega_{1}\right) \cosh \beta_{1} L\right]=0$

- The bending moment also is equal to the multiplying the effective mass moment of inertia at the cantilever beam tip $(\mathrm{J})$ with its rotational acceleration $(\alpha)$

$$
I E \frac{\partial^{2} w(L, t)}{\partial x^{2}}=-J \alpha
$$

Where $\quad \alpha=\frac{\partial^{3} w(L, t)}{\partial t^{2} \partial x}$

By substituting equations (3) into equation (9)

$$
\begin{aligned}
& C_{1}\left[-I E \beta_{1}^{2} \sin \beta_{1} L-\beta_{1} \omega_{1}^{2} J \cos \beta_{1} L\right]+ \\
& C_{2}\left[-I E \beta_{1}^{2} \cos \beta_{1} L+\beta_{1} \omega_{1}^{2} J \sin \beta_{1} L\right]+ \\
& C_{3}\left[I E \beta_{1}^{2} \sinh \beta_{1} L-\beta_{1} \omega_{1}^{2} J \cosh \beta_{1} L\right]+ \\
& C_{4}\left[I E \beta_{1}^{2} \cosh \beta_{1} L-\beta_{1} \omega_{1}^{2} J \sinh \beta_{1} L\right]=0
\end{aligned}
$$

And by rewriting equations (4), (5), (8), and (10) in a matrix form to solve them and find the unknown constants

$$
\left[\begin{array}{cccc}
0 & 1 & 0 & 1 \\
1 & 0 & 1 & 0 \\
S_{31} & S_{32} & S_{33} & S_{34} \\
S_{41} & S_{42} & S_{43} & S_{44}
\end{array}\right]\left[\begin{array}{l}
C_{1} \\
C_{2} \\
C_{3} \\
C_{4}
\end{array}\right]=\left[\begin{array}{l}
0 \\
0 \\
0 \\
0
\end{array}\right]
$$

Where:

$$
\begin{aligned}
& S_{31}=\left[-I E \beta_{1}^{3} \cos \beta_{1} L+\left(M_{t} \omega_{1}^{2}-C_{t} \omega_{1}\right) \sin \beta_{1} L\right] \\
& S_{32}=\left[I E \beta_{1}^{3} \sin \beta_{1} L+\left(M_{t} \omega_{1}^{2}-C_{t} \omega_{1}\right) \cos \beta_{1} L\right] \\
& S_{33}=\left[I E \beta_{1}^{3} \cosh \beta_{1} L+\left(M_{t} \omega_{1}^{2}-C_{t} \omega_{1}\right) \sinh \beta_{1} L\right] \\
& S_{34}=\left[I E \beta_{1}^{3} \sinh \beta_{1} L+\left(M_{t} \omega_{1}^{2}-C_{t} \omega_{1}\right) \cosh \beta_{1} L\right] \\
& S_{41}=\left[-I E \beta_{1}^{2} \sin \beta_{1} L-\beta_{1} \omega_{1}^{2} J \cos \beta_{1} L\right] \\
& S_{42}=\left[-I E \beta_{1}^{2} \cos \beta_{1} L+\beta_{1} \omega_{1}^{2} J \sin \beta_{1} L\right] \\
& S_{43}=\left[I E \beta_{1}^{2} \sinh \beta_{1} L-\beta_{1} \omega_{1}^{2} J \cosh \beta_{1} L\right]
\end{aligned}
$$

$S_{44}=\left[I E \beta_{1}^{2} \cosh \beta_{1} L-\beta_{1} \omega_{1}^{2} J \sinh \beta_{1} L\right]$

The characteristic equation of the system can be obtained as:

$$
\left|\begin{array}{cccc}
0 & 1 & 0 & 1 \\
1 & 0 & 1 & 0 \\
S_{31} & S_{32} & S_{33} & S_{34} \\
S_{41} & S_{42} & S_{43} & S_{44}
\end{array}\right|
$$

$=0$

The previous equation is used to obtain the natural frequency $\left(\omega_{1}\right)$ and from this equation, it is clear that the main parameters affecting the natural frequency are $\left(l, I, J, M_{t}, \rho\right.$, and $\left.\mathrm{E}\right)$.

\subsection{Harmonic Excitation Modelling 2.2.1. The transverse response of harmonic base excitation}

This section focuses on analyzing a forced vibration system with damping for determining the transverse displacement response with the time, and the power harvested from this investigation. The absolute transverse motion $\left(w_{t}(x, t)\right)$ of any point in the cantilever beam can be represented as the sum of the vibrator excitation motion $\left(w_{b}(t)\right)$ and the absolute cantilever beam displacement for the vibrator $(w(x, t))$. It should be noted here that when substitution in equation (1) which governs the free vibration equation of motion with the value of the absolute displacement, it turns into a forced vibration equation [11-13].

$$
E I \frac{\partial^{4} w(x, t)}{\partial x^{4}}+\rho A \frac{\partial^{2} w(x, t)}{\partial t^{2}}=-\rho A \frac{\partial^{2} w_{b}(x, t)}{\partial t^{2}}
$$

It is noticeable from the previous equation that the effective force resulting from the vibrator harmonic excitation which is represented in term $\left(-\rho A\left(\partial^{2} w_{b}(x, t) / \partial t^{2}\right)\right)$ is not a concentrated load, but equivalent to a distributed load of a mass $(\rho A)$ along the cantilever when moving with an acceleration $\left(\partial^{2} w_{b}(x, t) / \partial t^{2}\right)$. When the total tip mass $\left(M_{t}\right)$ is taken into consideration, the distributed mass $(\rho A)$ is replaced by $\left(\rho A+M_{t} \delta(x-L)\right)$, where $\delta(x-L)$ is a Dirac delta function [14]. Therefore, the equation of motion of the system becomes:

$$
\begin{aligned}
& E I \frac{\partial^{4} w(x, t)}{\partial x^{4}}+\rho A \frac{\partial^{2} w(x, t)}{\partial t^{2}} \\
& =-\left(\rho A+M_{t} \delta(x-L)\right) \frac{\partial^{2} w_{b}(x, t)}{\partial t^{2}}
\end{aligned}
$$

Perceiving that the excitation force is sinusoidal leads to conclude that the general solution of the previous system equation, which represents system response is also sinusoidal with time as: 
$w(x, t)=\mathrm{a}_{1} \Psi_{1}(x) \sin \Omega t-\mathrm{a}_{2} \Psi_{2}(x) \cos \Omega t$

whereas $\mathrm{a}_{1} \Psi_{1}(x)$ and $\mathrm{a}_{2} \Psi_{2}(x)$ represent trial functions that satisfy the problem boundary conditions, and $(\Omega)$ is the excitation frequency. Here the motion can be represented as two function combinations, the first one $\Psi_{1}(x)$ is in the same phase with the force function, and the second one $\Psi_{2}(x)$ is delayed by a 90-degree angle[15].

By considering that $\left(\Psi_{1}(x)=\Psi_{2}(x)=W(x)\right)$ and the vibrator excitation motion $\left(w_{b}(t)=w_{b} \sin \Omega t\right)$, the system equation of motion can be expressed as :

$E I \frac{d^{4} W(x)}{d x^{4}}\left[a_{1} \sin \Omega t-a_{2} \cos \Omega t\right]$

$+\rho A\left[-a_{1} \Omega^{2} \sin \Omega t+a_{2} \Omega^{2} \cos \Omega t\right] W(x)$

$=w_{b} \Omega^{2}\left(\rho A+M_{t} \delta(x-L)\right) \sin \Omega t$

Where the term $\left[w_{b} \Omega^{2}\left(\rho A+M_{t} \delta(x-L)\right)\right]$ is represented the amplitude of the excitation force $\left(F_{0}\right)$. The previous equation can be reformulated by separating the coefficients of $(\sin \Omega t)$ and $(\cos \Omega t)$, both in a separate equation. And by applying the Galerkin procedure to each of them by integrating them over the length $(L)$ of the continuous cantilever beam.

$$
\begin{aligned}
& \int_{0}^{L}\left[a_{1} E I \frac{d^{4} W(x)}{d x^{4}}-a_{1} \rho A \Omega^{2} W(x)\right] W(x) d x \\
& =\int_{0}^{L}\left[w_{b} \Omega^{2}\left(\rho A+M_{t} \delta(x-L)\right)\right] W(x) d x \\
& \int_{0}^{L}\left[a_{2} E I \frac{d^{4} W(x)}{d x^{4}}-a_{2} \rho A \Omega^{2} W(x)\right] W(x) d x
\end{aligned}
$$

After solving these equations and find the values of the constants $\left(a_{1}\right)$ and $\left(a_{2}\right)$, the absolute transverse amplitude $w_{t}(x, t)$ of the cantilever beam can be determined as:

$w_{t}(x, t)=\left[a_{1} W(x)+w_{b}\right] \sin \Omega t-$

$\left[a_{2} W(x)\right] \cos \Omega t \quad(19)$

$$
\left|W_{t}\right|=\sqrt{\left(a_{1} W(x)+w_{b}\right)^{2}+\left(a_{2} W(x)\right)^{2}}
$$

\subsubsection{Electrodynamic harvester modeling}

The relative oscillations of the electromagnetic energy harvester cause a variation in the magnetic flux and this creates an electromotive force $\left(V_{E M F}\right)$ in the coil and this voltage is proportional to the time-varying magnetic flux according to Faraday's law of induction [16-20].

$V_{E M F}=-B L_{w} v_{t}$
Where (B) is the magnetic field strength, $\left(L_{w}\right)$ is the total length of the coil wire, and $\left(v_{t}\right)$ is the velocity of the magnet and can be expressed as

$v_{t}=\frac{\partial w_{t}(x, t)}{\partial t}$

Upon substitution into equation (20), the velocity of the magnet can be determined as

$$
v_{t}=\Omega\left[a_{1} W(x)+w_{b}\right] \cos \Omega t+\Omega\left[a_{2} W(x)\right] \sin \Omega t
$$

When the magnet is placed at the free end of the cantilever beam at $(x=l)$, the speed of the magnet becomes:

$$
\left|v_{t}\right|=\Omega \sqrt{\left(a_{1} W(l)+w_{b}\right)^{2}+\left(a_{2} W(l)\right)^{2}}
$$

The induced current passing through the coil circuit can be written as:

$$
i(t)=\frac{V_{E M F}}{R_{l}+R_{c}+j \Omega L_{c}}
$$

Where $\left(R_{l}\right)$ is a load resistance, $\left(R_{c}\right)$ is the coil resistance, $(\Omega)$ is the excitation frequency, and $\left(L_{c}\right)$ is the inductance of the coil. At the low frequencies (less than $1 \mathrm{kHz}$ ) [17], the coil inductance term can be neglected and the coil resistance dominates its impedance. This the magnetic field will generate a magnetic force $\left(F_{m}\right)$ which has a reverse direction to the magnet movement direction.

$F_{m}=B L_{w} i(t)$

This electromagnetic force is proportional to the magnet velocity and it can be expressed also as the product of velocity with the electromagnetic damping $\left(C_{m}\right)$

$F_{m}=C_{m} \frac{\partial w_{t}(x, t)}{\partial t}$

From equations (25), (26), and (27) the electromagnetic damping can be given from the following equation:

$$
C_{m}=\frac{\left(B L_{w}\right)^{2}}{R_{l}+R_{c}}
$$

And the harvested power can be calculated as

$$
P=R_{l} i^{2}(t)=\frac{\left[B L_{w}\left(\partial w_{t}(x, t) / \partial t\right)\right]^{2} R_{l}}{\left(R_{l}+R_{c}\right)^{2}}
$$

\section{EXPERIMENTAL WORK}

Figure 3 shows a close-up view and the components used in the design of the experimental set-up of the 
electromagnetic energy harvester with the proposed tuning mechanism. A cantilever beam with length 60 $\mathrm{mm}$, width $28 \mathrm{~mm}$, and thickness $1 \mathrm{~mm}$ beam is mounted on a shaker (Brüel \& Kjær type 3386-062) to obtain and control the ambient excitation frequency whilst the tuning mechanism is attached with the other free end of it. The tuning mechanism consists of a movable tuning mass on a lead screw which derives its motion from a DC motor. two guide shafts fixed on two acrylic plates are used to convert a rotational motion of the motor into a linear motion for the proof mass. The main parameters and material properties of the proposed design are listed in Table 1.

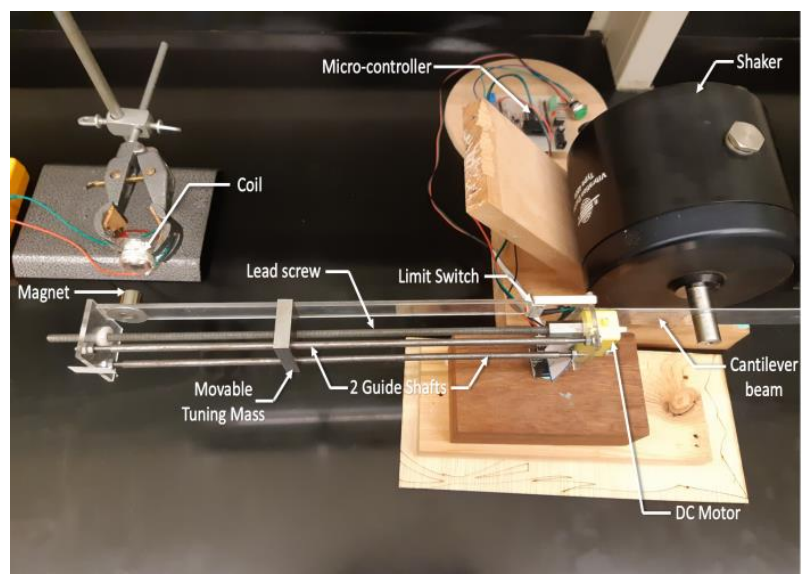

Figure 2 Close up view of the electromagnetic energy harvester with the proposed tuning mechanism

In the beginning, it is necessary to find the natural frequency value of the energy harvester at the different positions of the tuning mass. The proof mass position $\left(\mathrm{L}_{\mathrm{m}}\right)$ is measured from the free end of the cantilever beam every $0.01 \mathrm{~m}$ from $\mathrm{L}_{\mathrm{m}}=0.04 \mathrm{~m}$ up to $0.28 \mathrm{~m}$. To keep the system continuously in the resonance zone, the automatic tuning technique is used in which the external frequency is measured periodically and the position of the mass is adjusted accordingly so that the natural frequency of the system matches with the ambient frequency whatever change.

Table 1: The characteristic properties of the proposed design

\begin{tabular}{|l|l|}
\hline \multicolumn{1}{|c|}{ Parameter } & \multicolumn{1}{c|}{ Value } \\
\hline Cantilever beam density $(\rho)$ & $7850 \mathrm{Kg} / \mathrm{m}^{3}$ \\
\hline Modulus of elasticity of cantilever $(\mathrm{E})$ & $213 \mathrm{GPa}$ \\
\hline Movable tuning mass $(\mathrm{m})$ & $0.118 \mathrm{Kg}$ \\
\hline Mass of guide shafts $\left(\mathrm{m}_{\mathrm{g}}\right)$ & $0.049 \mathrm{Kg}$ \\
\hline Mass of screw $\left(\mathrm{m}_{\mathrm{s}}\right)$ & $0.056 \mathrm{Kg}$ \\
\hline DC motor mass $\left(\mathrm{m}_{\mathrm{m}}\right)$ & $0.06 \mathrm{Kg}$ \\
\hline Width of movable mass $(\mathrm{b})$ & $0.0095 \mathrm{~m}$ \\
\hline Screw length $\left(\mathrm{L}_{\mathrm{s}}\right)$ & $0.32 \mathrm{~m}$ \\
\hline Guide shafts length $\left(\mathrm{L}_{\mathrm{g}}\right)$ & $0.3 \mathrm{~m}$ \\
\hline Magnetic field strength $(\mathrm{B})$ & $1.3 \mathrm{Tesla}$ \\
\hline
\end{tabular}

At resonance state, the highest response is obtained and therefore this mechanical energy can be converted into electrical energy. In the proposed design, electromagnetic energy harvester is used by attaching a permanent magnet ( $\mathrm{NdFeB} \mathrm{N} 42)$ at the free end of the mechanism and placing a coil with inner diameter 0.04 $\mathrm{m}$, outer diameter $0.05 \mathrm{~m}$, and height $0.015 \mathrm{~m}$ on a separate holder in front of this magnet. The coil is connected to a decade resistance box and oscilloscope (Tektronix TDS2024C) which used to measure the output voltage from this energy harvester.

\section{RESULTS AND DISCUSSION}

The study of the analytical model shows that the most important parameters affecting on the natural frequency as explained in the characteristic equation of the system are the cantilever beam length $(L)$, its second moment of area $(I)$, the total mass of the tuning mechanism $\left(M_{t}\right)$, and the system mass moment of inertia $(J)$. A parametric study has been conducted to these factors which by modifying them can adjust the natural frequency, and this study was carried out on 3 positions of the movable tuning mass, which are: $0.04,0.15$, and $0.28 \mathrm{~m}$ from the free end of the cantilever beam.

The study has shown that the cantilever beam length is a very effective factor on the natural frequency, as any slight increase in its length leads to a clear decrease in the natural frequency of the system. Table 2 and Figure 3 shows the observed decrease in the natural frequency when changing the length of the cantilever beam from 0.04 to $0.17 \mathrm{~m}$.

Table 2: Influence of cantilever beam length on the natural frequency at different tuning mass positions

\begin{tabular}{|c|c|c|}
\hline \multirow{2}{*}{$\mathrm{L}_{\mathrm{m}}(\mathrm{m})$} & \multicolumn{2}{|c|}{$\omega_{n}(\mathrm{HZ})$} \\
\cline { 2 - 3 } & $\mathrm{L}=0.04 \mathrm{~m}$ & $\mathrm{~L}=0.17 \mathrm{~m}$ \\
\hline 0.04 & 5.133 & 1.42 \\
\hline 0.15 & 4.323 & 1.267 \\
\hline 0.28 & 3.377 & 1.091 \\
\hline
\end{tabular}

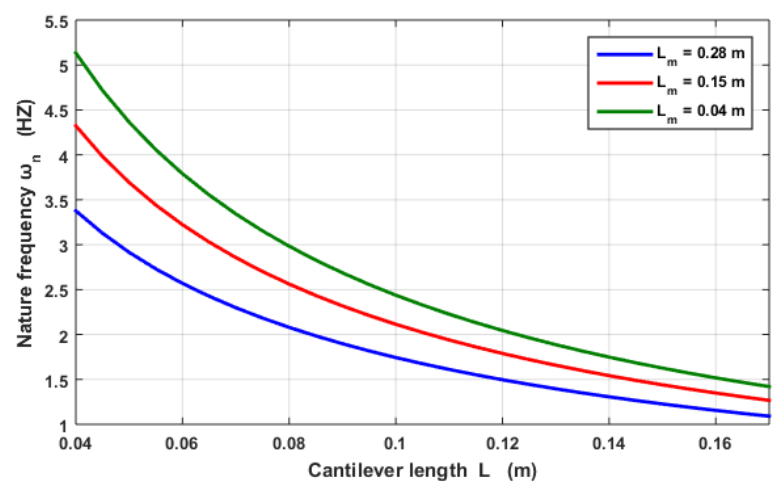

Figure 3: Effect of cantilever beam length on the natural frequency 
This study also dealt with the effect of the mass moment of inertia in more detail and attention where it was found that it is one of the most influencing factors on the natural frequency, so any increase in the mass moment of inertia, even a slight one, is accompanied by a noticeable decrease in the natural frequency, as illustrated in Figure 4. The mass moment of inertia can be changed by varying one of the parameters influencing it, such as weight, position, shape, or thickness of the effective mass.

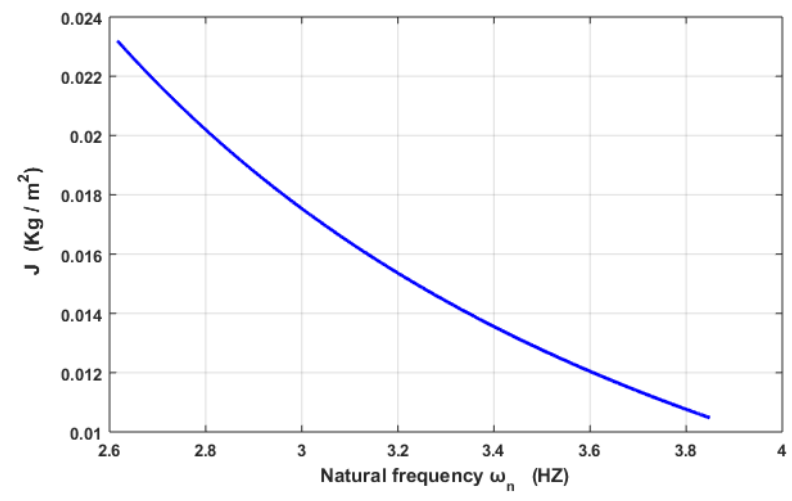

Figure 4: Effect of mass moment of inertia on the natural frequency

Figure 5 shows the effect of the movable tuning mass weight on the natural frequency, as when the weight of mass increases from 0.1 to $0.25 \mathrm{~kg}$, this leads to a noticeable decrease in the natural frequency. The percentages of decrease in the natural frequency vary according to the mass position, as these percentages decrease with increasing mass distance from the cantilever beam free end to become $7.8 \%, 20.2 \%$, and $30.2 \%$ at mass positions $0.04,015$, and $0.28 \mathrm{~m}$ respectively.

It was found that when studying the effect of mass geometry represented by its width, the natural frequency was not strongly influenced by increasing the mass width from 5 to $25 \mathrm{~mm}$, as the percentage of natural frequency decrease with the increase in the width was between 1.2:1.7 \% at the different tuning mass positions as illustrated in Figure 6. While it was found that changing the tuning mass distance from the cantilever beam free end has a great effect on the natural frequency, where it is inversely proportional to the tuning mass position as shown in Figure 7.

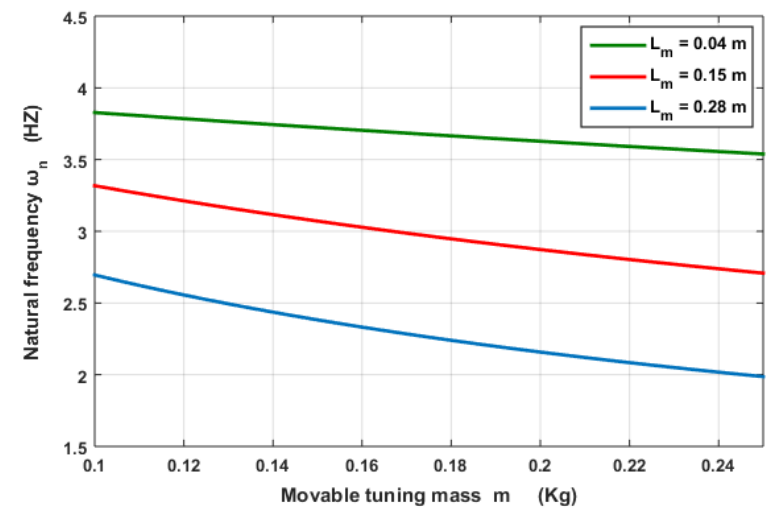

Figure 5: Effect of movable tuning mass weight on the natural frequency

Since the design idea is based on automatic resonance adjustment, the most suitable solution is to change the effective mass position to adjust the natural frequency and then reach the resonance state continuously. Upon verification of the theoretical results with the experimental results, great compatibility has appeared Where the natural frequency can be tuned practically from 2.65 to $3.75 \mathrm{~Hz}$ with tunability $38.4 \%$, whilst the tunability was $34.1 \%$ theoretically.

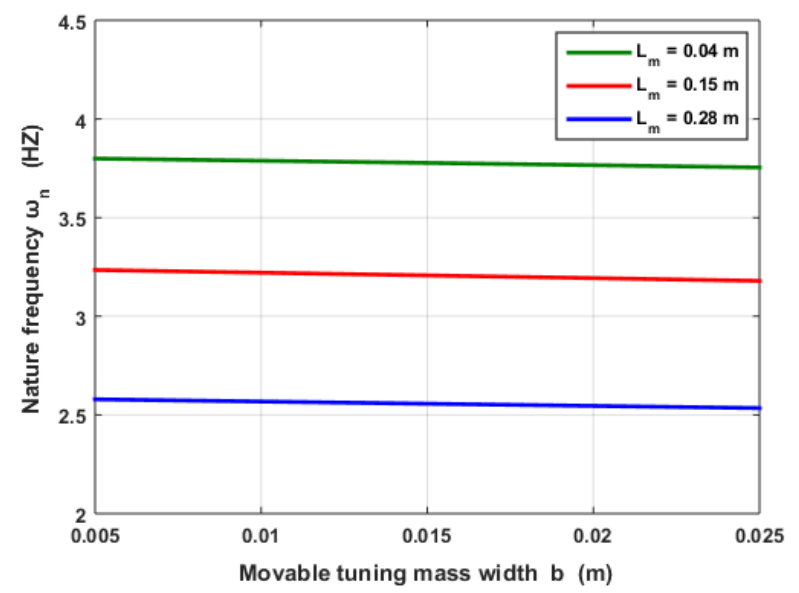

Figure 6: Effect of movable tuning mass width on the natural frequency

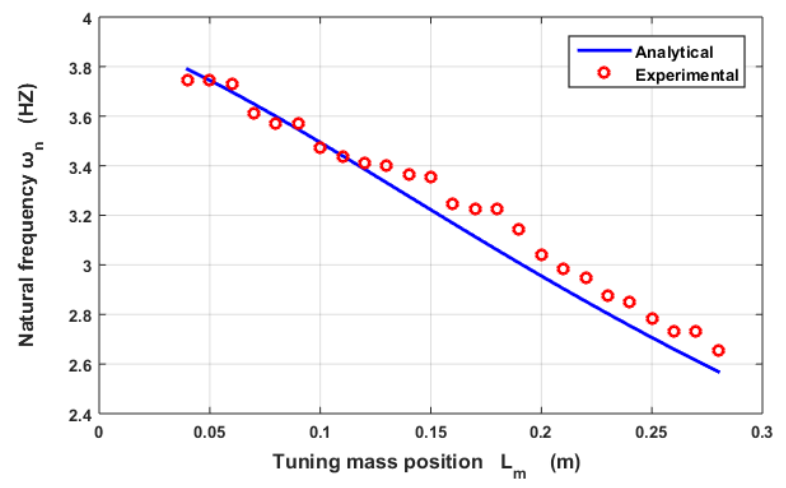

Figure 7: Effect of movable tuning mass position on the natural frequency 
The system response was calculated within the frequency range from 2 to $4.2 \mathrm{~Hz}$ theoretically and compared it with the experimental measurements at the mass position $0.04,0.15$, and $0.28 \mathrm{~m}$ respectively as described in Figure 8. The experimental results showed that the maximum system response in the resonance state depends on the tuning mass position, where the maximum response reduced by $18.4 \%$ at the mass position $\left(\mathrm{L}_{\mathrm{m}}=0.28 \mathrm{~m}\right)$ than it at $\left(\mathrm{L}_{\mathrm{m}}=0.04 \mathrm{~m}\right)$, whereas the analytical model results showed a great approaching with the practical results.

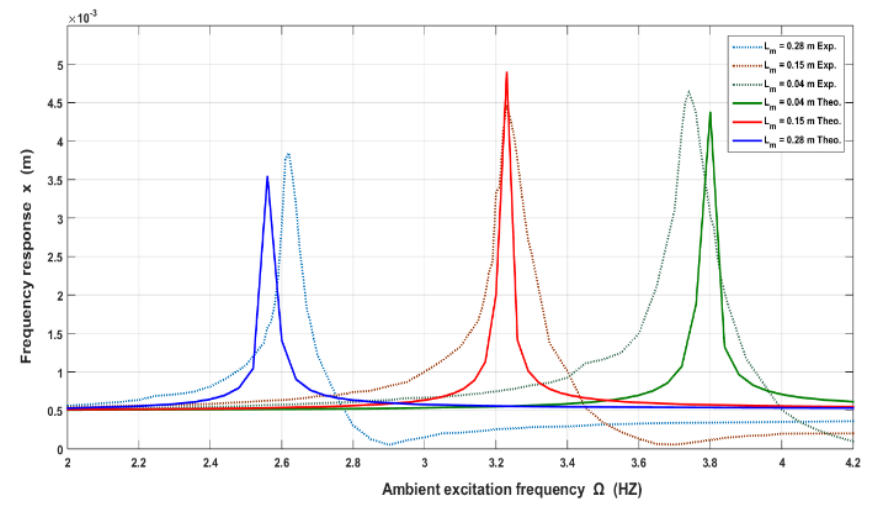

Figure 8: Effect of ambient frequency on response

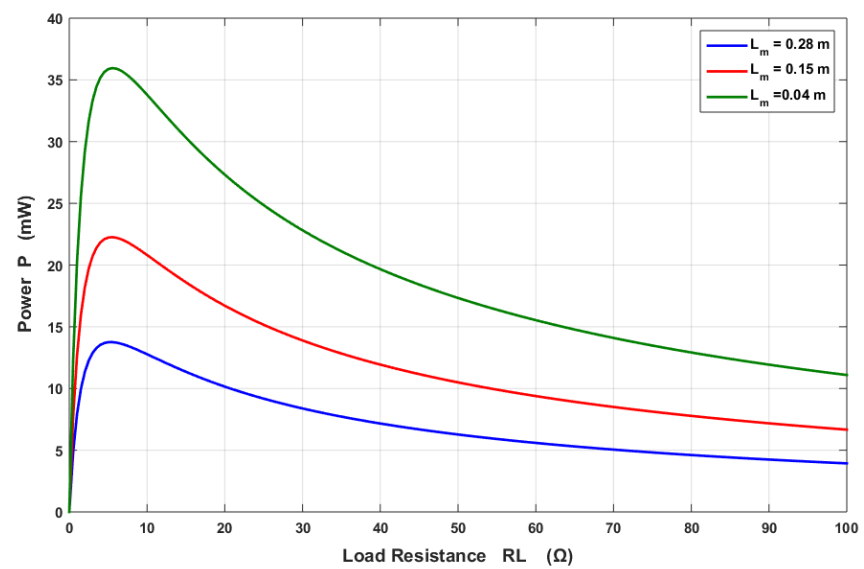

Figure 9: Effect of load resistance on output power

The power harvested was also calculated in the resonance state within the range of load resistance (RL) from 0 to $100 \Omega$, to obtain the optimum load resistance for each case, and accordingly harvest a large amount of energy as illustrated in Figures 9. It was noticed that the power harvested was increased with increasing the load resistance until it reached its maximum value at the optimum load resistance, after that the harvested power reduced again with increasing the load resistance. The position of the tuning mass also showed a significant influence on the output power in the resonance state, when the tuning mass distance $0.04,0.15$, and $0.28 \mathrm{~m}$ from the free end of the cantilever beam the harvested powers were $35.9,22.2,13.8 \mathrm{~mW}$ with optimum resistance load 6,5 , and $5 \Omega$ respectively.

\section{CONCLUSIONS}

The present investigation declares an innovative design of an automatic energy harvester system whose idea depends on adjusting the natural frequency of the system to match the ambient frequency. The presence of the system in resonance state continuously ensures harvesting the largest amount of energy. The power has been harvested using an electromagnetic energy harvester. The design is analyzed using the methodology of a vibration continuous system for investigating the different parameters of the tuning mechanism and verified experimentally.

The study proved that both cantilever beam length and mass moment of inertia is the most effective parameters on the system's natural frequency, as any slight increase in them leads to a clear decrease in the natural frequency of the system. It is also clarified that the best way to adjust the mass moment of inertia is to change the effective mass position. Otherwise, changing the proof mass's weight or its geometry was not sufficiently effective on the natural frequency, besides the difficulty of their automatic adjustment. The present study also indicates a great success of the proposed design in tuning the natural frequency experimentally within the range of 2.65 to $3.75 \mathrm{~Hz}$ with a tunability of $38.4 \%$. The proof mass position is modified automatically with any variation in the ambient frequency. The current investigation is suitable for harvesting energy from low frequencies where the harvested power reached 35.9 MW.

\section{Credit Authorship Contribution Statement}

Shahenda M. Attya: Methodology, Software, Formal analysis, Experimental work, Writing original draft. Ebtisam F. Abd-Gwad: Writing review \&editing, Supervision.

\section{Declaration of Competing Interest}

The authors declare that there is no conflict of interest regarding the publication of this paper.

\section{References}

[1] D. Zhu, "Vibration energy harvesting: machinery vibration, human movement, and flow-induced vibration," in Sustainable Energy Harvesting Technologies-Past, Present, and Future: InTech, 2011.

[2] D. Zhu, M. J. Tudor, and S. P. Beeby, "Strategies for increasing the operating frequency range of vibration energy harvesters: a review," Measurement Science and Technology, vol. 21, no. 2, 2010.

[3] S. W. Ibrahim and W. G. Ali, "A review on frequency tuning methods for piezoelectric energy harvesting systems," Journal of 
Renewable and Sustainable Energy, vol. 4, no. 6, 2012.

[4] P. J. Cornwell, J. Goethal, J. Kowko, and M. Damianakis, "Enhancing Power Harvesting using a Tuned Auxiliary Structure," Journal of Intelligent Material Systems and Structures, vol. 16, no. 10, pp. 825-834, 2005/10/01 2005.

[5] L. M. Miller, P. K. Wright, C. C. Ho, J. W. Evans, P. C. Shafer, and R. Ramesh, "Integration of a low frequency, tunable MEMS piezoelectric energy harvester and a thick film micro capacitor as a power supply system for wireless sensor nodes," in 2009 IEEE Energy Conversion Congress and Exposition, 2009, pp. 2627-2634: IEEE.

[6] X. Wu, J. Lin, S. Kato, K. Zhang, T. Ren, and L. J. P. o. P. Liu, "A frequency adjustable vibration energy harvester," pp. 245-248, 2008.

[7] J. Schaufuss, D. Scheibner, and J. Mehner, "New approach of frequency tuning for kinetic energy harvesters," Sensors and Actuators A: Physical, vol. 171, no. 2, pp. 352-360, 2011/11/01/ 2011.

[8] C. Williams, R. B. J. s. Yates, and a. A. Physical, "Analysis of a micro-electric generator for microsystems," vol. 52, no. 1-3, pp. 8-11, 1996.

[9] S. S. Rao, Mechanical Vibrations 5th Edition ed. 2004.

[10] S. S. Rao, Vibration of Continuous Systems, 1st edition ed. 2007.

[11] S. Timoshenko, D. Young, and W. J. I. Weaver, New York, "Vibration Problems in Engineering. John Willey and Sons," 1974.

[12] A. Erturk and D. J. Inman, "On mechanical modeling of cantilevered piezoelectric vibration energy harvesters," Journal of Intelligent Material Systems and Structures, vol. 19, no. 11, pp. 1311-1325, 2008.

[13] A. Erturk and D. J. Inman, "A distributed parameter electromechanical model for cantilevered piezoelectric energy harvesters," Journal of vibration acoustics, vol. 130, no. 4, p. 041002, 2008.

[14] A. Erturk, D. J. J. S. M. Inman, and Structures, "Issues in mathematical modeling of piezoelectric energy harvesters," vol. 17, no. 6 , p. 065016, 2008.

[15] A. J. J. o. S. Leissa and Vibration, "A direct method for analyzing the forced vibrations of continuous systems having damping," vol. 56, no. 3, pp. 313-324, 1978.

[16] D. Briand, E. Yeatman, and S. Roundy, Micro Energy Harvesting. Wiley-VCH Verlag GmbH \& Co. KGaA, 2015.

[17] S. Priya and D. J. Inman, Energy harvesting technologies. Springer, 2009.

[18] S. P. Beeby, M. J. Tudor, R. N. Torah, S. Roberts, T. O'Donnell, and S. Roy,
"Experimental comparison of macro and micro scale electromagnetic vibration powered generators," Microsystem Technologies, vol. 13, no. 11-12, pp. 1647-1653, 2007.

[19] M. M. R. El-Hebeary, M. H. Arafa, and S. M. Megahed, "Modeling and experimental verification of multi-modal vibration energy harvesting from plate structures," Sensors and Actuators A: Physical, vol. 193, pp. 35-47, 2013.

[20] C. Wei and X. Jing, "A comprehensive review on vibration energy harvesting: Modelling and realization," Renewable and Sustainable Energy Reviews, vol. 74, pp. 1-18, 2017. 